Mathematical Sciences And Applications E-Notes

Volume 3 No. 2 Pp. 99 (2015) (c)

\title{
ERRATUM TO :ESTIMATION USING COPULA FUNCTION IN REGRESSION MODEL. [MATHEMATICAL SCIENCES AND APPLiCATIONS E-NOTES, VOLUME 2, NO. 1, 2014, PAGES: 106-116]
}

\author{
DJAMILA BENNAFLA, ABBES RABHI, AMINA ANGELIKA BOUCHENTOUF
}

1. Along the Paper: instead of $h_{n}$ We Should have $a_{n}$.

2. On page 109, after this Sentence: (Now, let us Present Our estimated MODEL, THE REGRESSION FUNCTION $r(x)$, IS GIVEN AS FOLLOWS:) INSTEAD OF

$$
r(x)=Y c_{n}(F(x), G(y)), \quad|Y| \leq M, \quad Y, m \in \mathbb{R} .
$$

WE SHOULD HAVE

$$
r(x)=\int_{\mathbb{R}} y g(y) c(F(x), G(y))=\mathbb{E}\left(Y c_{n}(F(x), G(y))\right), \quad|Y| \leq M, \quad Y, m \in \mathbb{R} .
$$

3. ON THE SAME PAGE

THIS REGRESSION FUNCTION $r(x)$ IS ESTIMATED BY A FUNCTION

$\hat{r}=\mathbb{E}\left(Y \hat{c}_{n}(F(x), G(y))\right.$. INSTEAD OF

$\hat{r}(x)=Y \hat{c}_{n}(F(x), G(y)$.

3. On page 111, Theorem 4.1 We Should have Let $\hat{r}(x)=\mathbb{E}\left(Y \hat{c}_{n}(F(x), G(y))\right.$. INSTEAD OF $\hat{r}(x)=Y c_{n}(F(x), G(y)$.

Stochastic Models, Statistics and Applications Moulay Tahar University, Saida P.O.Box 138 En-Nasr Saida 20000 Algeria.

E-mail address: DBENNAFLA@YAHOO.FR

Department of Mathematics, Duillali liabes University, Sidi Bel Abbes, B.P. 89, Sidi Bel Abbes 22000, Algeria.

E-mail address: RABHI_ABBES@YAHOO.FR

Department of Mathematics Djillali liabes University of Sidi Bel Abbes, B.P. 89, Sidi Bel AbBes 22000, Algeria.

E-mail address: BOUCHENTOUF_AMINA@YAHOO.FR 